\title{
Wasser, Boden, Luft
}

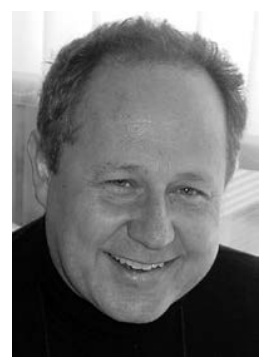

Manfred Wildner

\section{Korrespondenzadresse}

Prof. Dr. med. Manfred Wildner

Bayerisches Landesamt für Gesundheit und Lebensmittelsicherheit

Veterinärstraße 2

85764 Oberschleißheim

Manfred.Wildner@lgl.bayern.de

Bibliografie

DOI https://doi.org/10.1055/a-0893-5140

Gesundheitswesen 2019; 81: 380-381

(c) Georg Thieme Verlag KG Stuttgart · New York

ISSN 0941-3790
Schon das Hippokratische Gesamtwerk beschäftigt sich mit „Luft, Wasser und Ortslage/Boden “ als Determinanten der Gesundheit [1]. Eine Einteilung, welche die Jahrhunderte überdauert hat, z. B. als die von Johann Peter Frank geforderten „Medizinischen Topographien“, Vorläufer der heutigen Gesundheitsberichte [2]. Die 1923 so (um)benannte Preußische Landesanstalt für „Wasser-, Boden- und Lufthygiene“ („WaBoLu“) trug diesen Dreiklang nach Eingliederung als Institut in das Bundesgesundheitsamt weiter im Namen. Erst nach Eingliederung in das Umweltbundesamt (UBA) verlor sich dieser Dreiklang - nominal, nicht inhaltlich. Dort ist die Aufbauorganisation inzwischen unter Aspekten wie Nachhaltigkeit u. a. (öko)systemisch neu geordnet.

Die Bedeutung dieses Dreiklangs für die menschliche Gesundheit, individuell und auf Bevölkerungsebene, ist nicht zufällig. Dass Qualität und Verfügbarkeit von Wasser und Boden Grundbedingungen für menschliche Gesellschaften sind ist eine Erfahrung, welche die Menschheitsgeschichte begleitet hat: der reale Kollaps ganzer Zivilisationen wurde auch wissenschaftlich mit der Misswirtschaft von Wasser und Boden in Beziehung gesetzt [3]. Weitere Beispiele menschengemachter Öko-Katastrophen sind das Abgraben von Wasser im Kleinen wie auch im heutigen großen Stil gewaltiger Staudämme, z. B. an Nil und Mekong, oder subtiler die Unverkäuflichkeit von Ledererzeugnissen aus dem fernen Osten als Folge der über Bodenbelastungen in die tierische Nahrungskette eingetragenen Schadstoffe. Dass nicht jede „Brunnenvergiftung“ auch faktisch zutraf, ist ein weiteres leidvolles Kapitel im Umgang mit Umweltängsten: Furcht hat man, die Angst hat einen.

Wasser und Boden sind dem Menschen mehr oder minder unmittelbar zugänglich. Die Luft ist der auch ganz konkret nur schwer zu fassende Teil unserer natürlichen Umwelt. Sind es hier nicht vor allem die Umweltängste, die uns umtreiben? Ruß und Abgase waren zu Beginn der Industrialisierung noch gut sichtbar. Diese hergebrachten Gefahren scheinen heute durch Filteranlagen, neue Energiequellen, veränderte Produktionstechniken und Arbeitsschutzmaßnahmen zumindest in weiten Teilen der westlichen Welt gering, wobei sie teilweise auch auf andere Risikoträger (Stichwort Kernkraft) verlagert wurden.

Doch was ist „gering“ - haben wir es bei dieser Risikoeinschätzung nicht mit einer im Kern normativen Bewertung der Relevanz zu tun? Die Quantifizierung von Gefährdungen, Schaden und Nutzen mag noch wissenschaftlich-objektivierbar nachzuvollziehen sein, die Bewertung als Risiko ist bereits ein normativer Vorgang, der sich in den zu treffenden Maßnahmen als vielschichtiger fachlich-politischer Prozess fortsetzt. War im London des beginnenden 20. Jahrhunderts noch der sich anhäufende Pferdemist die drängendste verkehrsbezogene Herausforderung, sind wir am Beginn des 21. Jahrhunderts mit den Folgen von Verbrennungsmotoren konfrontiert. In der Analyse des Soziologen Ulrich Beck hat sich eine moderne Risikogesellschaft herausgebildet: „die Verteilungsprobleme und -konflikte der Mangelgesellschaft [werden] überlagert durch die Probleme und Konflikte, die aus der Produktion, Definition und Verteilung wissenschaftlich-technisch produzierter Risiken entstehen" [4, S. 25].

Wissen schafft paradoxerweise zugleich Gewissheiten und Unsicherheiten. In Anerkennung dieser Doppelgesichtigkeit können durch wissenschaftliche Studien Evidenzen in verschiedenen Wissenschaftszweigen erzeugt werden. Diese können in einer übergreifenden Abwägung ihren ersten Niederschlag in Empfehlungen zu Richtwerten finden, wie sie z. B. von der Weltgesundheitsorganisation vorgeschlagen werden. Für eine Festlegung von verbindlichen Grenzwerten z. B. als Richtlinie der Europäischen Union ist jedoch das nochmalige, auch politische Überdenken von Schadstoffen und Schadstoffgemischen, Gesundheitseffekten und verwundbaren Bevölkerungsgruppen, Unsicherheiten der Evidenz und gebotener Vorsorge, realistischen Möglichkeiten der Vorsorge und Akzeptabilität von Restrisiken vonnöten [5].

An dieser Stelle sei eine entschiedene Position für die selbst stille Materie „Luft“ ergriffen, mit einer Analogie zwischen unserem planetaren Ökosystem und dem menschlichen Organismus. Der verletzlichste Teil unserer Körperoberfläche ist die im Körper verund geborgene Lunge: 300 Millionen Lungenbläschen, welche auf 
einer Oberfläche von etwa 100 qm den lebensnotwendigen Gasaustausch mit der uns umgebenden Innen- und Außenluft ermöglichen. In ähnlicher Funktion kann auch unsere Atmosphäre betrachtet werden: als das Atmungsorgan unseres Planeten, einem verletzlichen Vermächtnis der Pflanzenwelt an die nachfolgenden Lebensformen, welches wie Wasser und Boden einer guten „Stewardship“ [6] bedarf. Nach Schätzungen der Weltgesundheitsorganisation (WHO) sind ein Viertel der menschlichen Krankheitslast umweltbedingt [7]. Namhafte Wissenschaftsorganisationen sprechen von einer „planetaren Gesundheit“ in einem inzwischen vom Menschen gestalteten neuen Erdzeitalter, dem Anthropozän [8].

„Stewardship“ ist dabei ein anspruchsvoller Begriff, der seinen Ursprung in der jüdisch-christlichen Schöpfungsgeschichte hat: den Auftrag an den Menschen, die Schöpfung zu hüten und zu bewahren [9]. Er umfasst einen systemischen und strategischen Governance-Ansatz, welcher Gesundheit in allen Politikfeldern thematisiert („Health in all policies“) und die kluge Balancierung verschiedener Einflussnahmen und Anforderungen erstrebt. Globales Denken - „think globally, act locally“ - ist hier ebenso gefragt wie ein bewusstes „One Health“-Konzept, welches die menschliche Gesundheit zusammen mit der Gesundheit von Tieren und dem umgebenden Ökosystem denkt. Um solche „gute Stewardship“ konkret zu machen: In der Diskussion um Luftschadstoffe greift eine Fokussierung auf einzelne Schadstoffe wie NOx mit Abgrenzung zu anderen Schadstoffen wie z. B. Feinstaub zu kurz [10]. Gleichermaßen kann die Regulierung einzelner Schadstoffe bei derart komplexen Problemlagen schnell zu „schrägen“ Folgeeffekten führen. Negative Folgen sind z. B. paradoxe Vermeidungsstrategien mit gesundheitlich kontraproduktiven Effekten wie einer Verkehrsverlagerung in Wohngebiete.

Notwendig sind angemessen komplexe Methoden sowohl in die Risikoanalyse wie auch bei den Bewältigungsstrategien [11]. Ein Beispiel dafür sind Gesundheitsfolgeabschätzungen (Health Impact Asessments, HIA) mit strukturierten Bewertungen auch der Abhilfestrategien [12]. Solche integrativen Bewertungen sind nicht der Individualmedizin und auch nicht der Epidemiologie alleine zuzuordnen, sondern gehören in den transdisziplinären Bereich Öffentlicher Gesundheit/Public Health. Sie verlangen eine entsprechende Forschungs- und Beratungsinfrastruktur, u. a. in Form von Schools of Public Health zur Evidenzfindung, Evidenzvermittlung, Beratung und Lehre, als Teil einer guten Stewardship gerade bei komplexen Zusammenhängen [13].

Auch die Berichte in diesem Heft wollen wieder Beiträge für solche gute Stewardship liefern. Sie befassen sich mit Arbeitsbedingungen und psychischer Gesundheit bei Ärztinnen und Ärzten, Fehlern und Schwächen in der Berichterstattung über epidemiologische Studien, einem epidemischem Vitamin D-Mangel bei weiblichen Inhaftierten, Substanzmissbrauch im Einzugsbereich des Giftnotrufes Erfurt 2002-2016, Stadtteildeprivation und Lebensmittelumwelt, dem Gesundheitsbeirat in München, der Zukunft der regionalen Versorgung in Deutschland, den Präferenzen junger Zahnärztinnen und Zahnärzte und den Amputationen der unteren Extremität. Last but not least auch mit einer methodischen Diskussion zur Berechnung vorzeitiger Todesfälle durch Umwelteinflüsse als Leserzuschrift und Replik der Autoren.

Die hippokratischen Schriften stellen in ihrem Nachdenken über Wasser, Boden und Luft die Jahreszeiten, die Winde und das Klima an die erste Stelle. Für eine darüber hinaus reichende Weisung sind wir allerdings auf unsere Jetztzeit angewiesen und auf die uns zur Verfügung stehenden, in der Menschheitsgeschichte einmaligen Erkenntnisquellen und Handlungsmöglichkeiten. Es geht dabei nicht so sehr um Messwerte und Grenzwerte, sondern um den vernünftigen Umgang mit ihnen, um vorsorgende und zugleich realitäts- und praxisnahe Abwägungen und auch um demokratisch legitimierte Entscheidungsprozesse. Ulrich Beck betrachtet dieses Nachdenken über die Folgeprobleme der technisch-ökonomischen Entwicklung als „Reflexiv“-Werden der Modernisierung [4]. Hans Jonas hatte schon früher mit seinem „Prinzip Verantwortung“ eine Ethik für die technologische Zivilisation versucht: „Handle so, dass die Wirkungen deiner Handlung verträglich sind mit der Permanenz echten menschlichen Lebens auf Erden“ [14]. Vernünftig ist beides und nicht nur „Wasser, Boden, Luft“ werden uns reflexiv-umsichtiges Handeln danken - auch unsere Kinder, Enkel und Urenkel.

\section{Literatur}

[1] Hippocrates. On airs, water, and places (Tanslation by Francis Adams). URL http://classics.mit.edu/Hippocrates/airwatpl.html download 10.02.2019

[2] Kuhn J. Die historische Entwicklung der kommunalen Gesundheitsberichterstattung - ein Forschungslücke. Gesundheitswesen 2007; 69 : 507-513

[3] Diamond J. Collapse: How Societies Choose to Fail or Succeed. New York: Penguin Group; 2005

[4] Beck U. Risikogesellschaft. Auf dem Weg in eine andere Moderne. Frankfurt a. M.: Suhrkamp; 1986

[5] Zylka-Menhorn V, Grunert D. Die Krux mit den Grenzwerten. Dt Ärzteblatt 2019; 116: C172-C173

[6] World Health Organization. Stewardship. https://www.who.int/ healthsystems/stewardship/en/ Zugang 2019/02/11

[7] Prüss-Ustün A, Wolf J, Corvalan C, Neville T, Bos R, Neira M. Diseases due to unhealthy environments: an updated estimate of the global burden of disease attributable to environmental determinants of health. J Public Health 2017; 39: 464-475

[8] Whitmee S, Haines A, Beyrer C et al. Safeguarding human health in the Anthropocene epoch: report of The Rockefeller Foundation-Lancet Commission on planetary health. Lancet 2015; 386: 1973-2028

[9] Saltman RB, Ferroussier-Davis O. The concept of stewardship in health policy. Bulletin World Health Org 2000; 78: 732-739

[10] Nationale Akademie der Wissenschaften Leopoldina. Saubere Luft. Stickstoffoxide und Feinstaub in der Atemluft: Grundlagen und Empfehlungen. Halle (Saale): 2019

[11] Haines A. Health in the Anthropocene Epoch - implications for epidemiology. Int J Epidemiol 2018: 47: 17271729:

[12] Wismar M, Blau J, Ernst K, Figueras ]., (Hrsg.). Die Wirksamkeit von Gesundheitsfolgenabschätzung. Umfang und Grenzen der Unterstützung von Entscheidungsprozessen in der Europäischen Region.Kopenhagen: WHO; 2007. http://www.euro.who.int/ document/E90794.pdf Zugang am 11.02.2019

[13] Dragano N, Gerhardus A, Kurth BM et al. Public Health - mehr Gesundheit für alle. Ziele setzen - Strukturen schaffen - Gesundheit verbessern. Gesundheitswesen 2016: 78: 686-688

[14] Jonas H. Das Prinzip Verantwortung. Versuch einer Ethik für die technologische Zivilisation.Frankfurt a. M.: Suhrkamp; 1979 S. 36 